\title{
DIVISION ALGEBRAS OVER AN ALGEBRAIC FIELD*
}

\author{
BY A. A. ALBERT
}

1. Introduction. $\mathrm{H}$. Hasse has given a rigorous treatment of the theory of quadratic null forms over the field $R$ of all rational numbers. He used the theory of $p$-adic numbers and readily extended his methods to obtain complete results on quadratic forms over any algebraic number field $\dagger$ and hence, by a simpleisomorphism, over any field $R(\theta)$, where $\theta$ is any quantity satisfying an equation with coefficients in $R$ and irreducible in $R$. Hasse has also used the fundamental principle of his quadratic form theory to prove several important theorems on cyclic (Dickson) algebras. $\ddagger$

I have recently obtained theorems on rational division algebras by the use of A. Meyer's theorem that every indefinite quadratic form, with rational coefficients, in five or more variables is a null form. $\S$ But now Hasse's theorems make the extension to algebras over algebraic fields $R(\theta)$ almost immediate. In particular it is shown here that the direct product of any two generalized quaternion algebras over $R(\theta)$ is never a division algebra,\| and that a sufficient condition that a normal division algebra of order sixteen over $R(\theta)$ be a cyclic algebra is that it contain a quantity $x$ not in $R(\theta)$ such that $x^{2}=\Delta_{1}^{2}+\Delta_{2}^{2}$ with $\Delta_{1}$ and $\Delta_{2}$ in $R(\theta)$.

* Presented to the Society, September 9, 1931.

† See his Darstellbarkeit von Zahlen durch quadratische Formen in einem beliebigen algebraischen Zahlkörper, Journal für Mathematik, vol. 153 (1923), pp. 113-130. These theorems seem to be not so well known in America as they should be. References on quadratic null forms are always made to the clumsy nineteenth century treatment of $\mathrm{A}$. Meyer and the partial reproductions of Bachmann instead of to the modern short rigorous $p$-adic discussion of Hasse. Moreover Hasse's extensions to algebraic fields are of extreme importance and they should be better known.

$\ddagger$ Theory of cyclic algebras over an algebraic number field, which will appear in the January, 1932, issue of the Transactions of this Society.

$\S$ For references see $\$ 3$.

\| This theorem has several applications. I shall use it in a paper to be offered for publication to the Transactions of this Society to prove that all normal division algebras of order sixteen over a field $R(\theta)$ are cyclic (Dickson) algebras. 
Hasse's new theorems on cyclic algebras are also used here to obtain an alternative proof of the above theorem on generalized quaternion algebras. In fact I show here that a necessary and sufficient condition that a direct product of any two normal division algebras over a field $R(\theta)$ be a division algebra is that their orders be relatively prime.

2. Preliminary Theorems. Let $F$ be any non-modular field. We shall assume the following known theorems.*

THEOREM 1. The order of any normal simple algebra is the square of an integer $n$ called the degree of the algebra. (WEDDERBURN.)

Theorem 2. Let $A$ be a simple algebra over $F$ and let $B$ be a normal simple sub-algebra of $A$. Then $A$ is the direct product $B \times C$ of $B$ and another simple sub-algebra $C$ of $A$. (WEDDERBURN.)

THEOREM 3. Every simple algebra $A$ over $F$ is expressible in the form $A=M \times D$ where $M$ is a total matric algebra and $D$ is a division algebra in one and only one way in the sense of equivalence, and conversely. (WEDDERBURN.)

In the following theorems we take $D$ to be a normal division algebra of order $m^{2}$, degree $m$, over its centrum $F$.

THEOREM 4. The grade $s$ of any quantity $y$ of $D$ is a divisor of the degree $m$ of $D$ and there exists a quantity $x$ in $D$ of grade $m$ with respect to $F$ such that $y$ is in $F(x)$. (AlBERT.)

THEOREM 5. Every root in $D$ of the minimum equation of $a$ quantity $y$ in $D$ is a transform $z^{-1} y z$ of $y$ by a quantity $z$ in $D$. (Albert.)

Theorem 6. The only quantities of $D$ commutative with $x$ in $D$ of grade $m$ with respect to $F$ are quantities of $F(x)$. (Dickson.)

Definition. An algebraic field $Z$ over $F$ is called a splitting field (Zerfällungskörper) of $D$ if $D \times Z$ is a total matric algebra.

* For proofs of the very well known Theorems 1, 3, 6, see L. E. Dickson's Algebren und ihre Zahlentheorie. For Wedderburn's Theorem 2, see the Proceedings of the Edinburgh Mathematical Society, vol. 25 (1906), pp. 1-3. For Theorem 4, see the author's Theorem 37 of his paper, Annals of Mathematics, vol. 30 (1929), pp. 583-590, and for Theorem 5, see the same journal, vol. 30 (1929), pp. 322-338, Theorem 12. Finally for proofs of Theorems 7, 8, 11, 12 see the author's paper, On direct products, Transactions of this Society, vol. 33 (1931), pp. 690-711. 
TheOREM 7. Let $Z$ be a splitting field of order $n$ over $F$ of $D$ of degree $m$ over $F$. Then $n=r m$, and if $M$ is a total matric algebra of degree $r$ the normal simple algebra $M \times D$ of degree $n$ over $F$ contains a sub-field $F(z)$ isomorphic to $Z$. (AlBert.)

THEOREM 8. Let $Y$ be an algebraic field of order $n$ over $F$. Then $A \times Y=M \times B$, where $M$ is a total matric algebra of degree s, and $B$ is a normal division algebra of degree $t$ over $Y$ such that $m=s t$, $n=s r$, so that $s$ divides $n$. (AlBeRT.)

Suppose that $y$ in $D$ has grade $s$ with respect to the centrum $F$ of $D$. By Theorem 4 we have $n=s t$ and there exists a quantity $x$ in $D$ of grade $m$ for $F$ such that $y$ is in $F(x)$. The algebraic field $F(x)$ is then a relative field of order $t$ over $F(y)$. If $\xi$ is a scalar root of the minimum equation of $x$ for $F$ then, as is very well known, $F(\xi)$ is a splitting field for $D$. If the expression for $y$ in $F(x)$ is $y=f(x)$, the scalar $\eta=f(\xi)$ has grade $s$ for $F$ and $F(\xi)$ is a relative field $K(\xi)$ of order $t$ over $K=F(\eta)$. Also $\eta$ is a scalar root of the minimum equation of $y$ for $F$. If $D^{\prime}=D \times K$, then $D^{\prime \prime}=D^{\prime} \times K(\xi)=D \times F(\xi)$ is a total matric algebra. But $D^{\prime}=M \times B$ where $M$ is a total algebra of degree $\alpha$ and $B$ is a normal division algebra of degree $\beta$ over its centrum $K$, such that

$$
m=\alpha \beta, s=\rho \alpha .
$$

Also $D^{\prime \prime}=D^{\prime} \times K(\xi)=M \times B \times K(\xi)$ is a total matric algebra so that, by Theorem 8 applied to $B$, the integer $\beta$ divides $t$. But $s t=m=\alpha \beta$ and $\alpha$ divides $s, \beta$ divides $t$. Hence $\alpha=s, \beta=t$. If $\eta_{0}$ is any other scalar root of the minimum equation of $y$ with respect to $F$, then $D \times F\left(\eta_{0}\right)$ is simply isomorphic with $D \times F(\eta)$ and has exactly the same properties. We have therefore proved the following theorem.

THEOREM 9. Let $y$ in $D$ have grade $s$ for $F$ so that $m=s t$. Then, if $\eta$ is any scalar root of the minimum equation of $y$ for $F$,

$$
D \times F(\eta)=M \times B,
$$

where $M$ is a total matric algebra of degree $s$ and $B$ is a normal division algebra of degree $t$ over its centrum $F(\eta)$.

Let $H$ be a total matric algebra equivalent to $M$ of Theorem 9 . As the author has shown (On direct products, loc. cit.) there ex- 
ists a quantity $\eta$ in $H$ satisfying the condition of Theorem 9 so that $D \times H$ contains $D \times F(\eta)$ as a sub-algebra. But $D \times F(\eta)$ $=M \times B$, so that, by Theorem $2, H \times D=M \times C$, where $C$ is necessarily a normal simple algebra. It follows from the uniqueness in Theorem 3 that $C$ is equivalent to $D$. Algebra $C$ is the algebra of all quantities of $H \times D$ which are commutative with all of the quantities of $M$, and contains $B$ as a sub-algebra. If $y_{1}$ in $D$ corresponds to $\eta$ in $C$ under the isomorphism between $D$ and $C$, then $y_{1}$ is a root of the minimum equation of $y$ with respect to $F$ and, by Theorem 5 , is a transform $z^{-1} y z$ of $y$ by a quantity $z$ in $D$. If the correspondence between the quantities of $D$ and $C$ is designated by $d \cong c$, then we can obviously set up a new simple isomorphism $z d z^{-1} \cong c$ in which now $y$ corresponds to $\eta$. Hence $D$ contains an algebra $B_{0}$ which is a normal division algebra of degree $t$ over its centrum $F(y)$ equivalent to $B$ as over $F(\eta)$. The algebra $G$ of all quantities of $D$ commutative with $y$ has $B_{0}$ as a sub-algebra and, by Theorem $2, G=B_{0} \times Q$, where $Q$ is an algebra over $F(y)$. By Theorem 4 algebra $B_{0}$ contains a quantity $b$ of grade $t$ with respect to $F(y)$. The field $F(b, y)$ has order st over $F$ and contains a quantity $x$ of grade $m$ with respect to $F$ generating it. But $x$ is in $B_{0}$ and is commutative with all of the quantities of $Q$. By Theorem 6 the quantities of $Q$ are in $F(x)$, and hence in $B_{0}$, so that $B_{0}$ is $G$.

THEOREM 10. The algebra $B$ of Theorem 9 is simply isomorphic with the algebra of all quantities of $D$ commutative with $y$, under $a$ correspondence where y corresponds to $\eta$. Hence this latter algebra is a normal division algebra of degree $t$ over its centrum $F(y)$.

We shall also use the following theorem of Brauer which I discovered independently and which I have proved in a short new way in my paper On direct products, loc. cit.

THEOREM 11. Write $m=p_{1}^{e_{1}} \cdot p_{2}^{e_{2}} \cdots p_{t}^{e_{t}}$, where the $p_{i}$ are distinct primes. Then $D=D_{1} \times D_{2} \times \cdots \times D_{t}$, where $D_{i}$ is a normal division algebra of degree $p_{i}^{e_{i}}$ over $F$ in one and only one way in the sense of equivalence, and conversely. (BRAUER, ALBERT.)

We require in addition the following theorem of my own.

Theorem 12. Let $m=p^{e}, p$ a prime, and let $x$ in $D$ have grade $m$ for $F$. Then there exists an algebraic field $Z$ of order $n$ over $F$, such that $n$ is prime to $p, D \times Z$ is a normal division algebra of de- 
gree $m$ over its centrum $Z$, and $Z(x)$ is a cyclic field of order $p$ over a sub-field $Z(y)$ of order $p^{e-1}$ over $Z$. (ALBERT.)

The following statement is an immediate consequence of Theorem 12, the definition of a cyclic algebra and Theorem 10 .

Theorem 13. Let $D^{\prime}=D \times Z, y$ and $x$ be as in Theorem 12 . Then the algebra $B$ of all quantities of $D^{\prime}$ commutative with $y$ is a cyclic algebra of degree $p$ over its centrum $Z(y)$.

3. Applications of the Hasse Theory of Quadratic Forms. Let $R$ be the field of all rational numbers, and let $\phi(\xi)=0$ with coefficients in $R$ and degree $m$ be irreducible in $R$. Let

$$
\xi_{1}, \cdots, \xi_{r}, \eta_{1}, \cdots, \eta_{s}, \bar{\eta}_{1}, \cdots, \bar{\eta}_{s}
$$

be the $m=r+2 s$ complex roots of the equation $\phi(\xi)=0$, where $\xi_{1}, \cdots, \xi_{r}$ are all real and $\eta_{1}, \cdots, \eta_{s}$ are all imaginary. Suppose that $\theta$ is any quantity such that $\phi(\theta)=0$ so that we can define an algebraic field $R(\theta)$ of order $m$ over $R$, which is evidently simply isomorphic with each of the algebraic number fields generated by each of (1). An $n$-ary quadratic form

$$
Q(\theta) \equiv Q\left(\theta ; x_{1}, \cdots, x_{n}\right) \equiv \sum_{i, j}^{1, \cdots, n} a_{i j}(\theta) x_{i} x_{j}
$$

with coefficients in $R(\theta)$ is called a null form if there exist $x_{1}, \cdots, x_{n}$ not all zero in $R(\theta)$ such that $Q=0$. Hasse has proved that an n-ary quadratic form (2), $n \geqq 5$, is a null form if and only if the corresponding real forms

$$
Q_{k} \equiv Q\left(\xi_{k}\right)=\sum_{i, j}^{1, \cdots, n} a_{i j}\left(\xi_{k}\right) x_{i} x_{j}, \quad(k=1, \cdots, r),
$$

are all indefinite.

We apply Hasse's theorem first to the form

$$
Q=\alpha x_{1}^{2}+\beta x_{2}^{2}-\alpha \beta x_{3}^{2}-\left(\rho x_{4}^{2}+\sigma x_{5}^{2}-\sigma \rho x_{6}^{2}\right) .
$$

For a fixed $k$, if we use the notation $\lambda\left(\xi_{k}\right)=\lambda_{k}$, then in $Q_{k}$ the numbers $\alpha_{k}, \beta_{k},-\alpha_{k} \beta_{k}$ all have the same sign only when all are negative. The numbers $-\rho_{k},-\sigma_{k}, \sigma_{k} \rho_{k}$ are never all negative, so that the forms $Q_{k}$ are all indefinite. Hence the form (3) is always a null form. I have proved* that if $B$ and $C$ are any two generalized quaternion algebras

* This Bulletin, vol. 37 (1931), pp. 301-312, in particular, p. 311. 


$$
\begin{aligned}
& B=(1, i, j, i j), \quad i^{2}=\alpha, \quad j^{2}=\beta, \quad j i=-i j, \\
& C=(1, I, J, I J), \quad I^{2}=\rho, \quad J^{2}=\sigma, J I=-I J,
\end{aligned}
$$

then the direct product $B \times C$ is a division algebra if and only if the form (3) is not a null form. But when $B$ and $C$ are algebras over $R(\theta)$ we have shown that (3) is always a null form. Hence we have proved the following result.

THEOREM 14. Let $B$ and $C$ be generalized quaternion algebras over a field $R(\theta)$. Then $B \times C$ is not a division algebra.

In a recent paper* $I$ proved that a sufficient condition that a normal division algebra $D$ of order sixteen over $R$ be a cyclic (Dickson) algebra, is that $D$ contain a quantity $x$ not in $R$ but such that $x^{2}=\Delta_{1}^{2}+\Delta_{2}^{2}$ with $\Delta_{1}$ and $\Delta_{2}$ in $R$. The proof was rational throughout except for the use of the analog to Theorem 14 on page 184, which could have been avoided, and the essential proof that the form numbered (53) was a null form. The proof was exactly of the same nature as our proof that (3) is a null form, depending only on intrinsic signs of coefficients, and is valid for any field $R(\theta)$. We may state then the validity of the following theorem without further proof.

Theorem 15. A sufficient condition that a normal division algebra $D$ of order sixteen over $R(\theta)$ be a cyclic (Dickson) algebra is that $D$ contain a quantity $x$ not in $R(\theta)$ such that $x^{2}=\Delta_{1}{ }^{2}+\Delta_{2}{ }^{2}$, where $\Delta_{1}$ and $\Delta_{2}$ are in $R(\theta)$.

I have recently published two papers, $\dagger$ of which the results and the proofs given are again valid for any field $R(\theta)$, except that Theorem 4 of the latter paper, where the Meyer theorem on quaternary quadratic null forms is applied, should be replaced by the Hasse criteria for quaternary quadratic forms over an algebraic number field isomorphic with $R(\theta)$.

We shall now pass to a generalization and alternative proof of Theorem 14.

4. The Direct Product $D \times B$. Let $A$ be any normal simple algebra over an algebraic field $F=R(\theta)$. By Theorem 3, $A=M \times D$,

* Transactions of this Society, vol. 32 (1930), pp. 171-195.

$\dagger A$ necessary and sufficient condition for the non-equivalence of any two rational generalized quaternion division algebras, this Bulletin, vol. 36 (1930), pp. 535-540, and $A$ construction of all non-commutative rational division algebras of order eight Annals of Mathematics, vol. 31 (1930), pp. 567-576. 
where $M$ is a total matric algebra and $D$ is a normal division algebra, the division algebra component of $A$. Hasse in his paper on cyclic algebras (loc. cit.) has called two normal simple algebras similar if they have the same division algebra components. Hasse has called a normal simple algebra cyclically representable if it is similar to a cyclic algebra and has proved the following theorems.

THEOREM 16. Every cyclically representable algebra $D$ over $R(\theta)$ is a cyclic algebra. (HASSE.)

THEOREM 17. The direct product of two cyclically representable algebras is cyclically representable. (HASSE.)

We also use the following known theorem (On direct products, loc. cit.).

TheOREM 18. Let $D$ be any normal division algebra over a nonmodular field. Then there exists an integer $\rho$ which divides the degree $m$ of $D$, such that $\rho$ is the least integer $\alpha$ for which the direct product $D^{\alpha}$ is a total matric algebra. In particular $D^{m}$ is a total matric algebra. (BRAUER, AlBeRT.)

The integer $\rho$ is called the exponent of $D$. Hasse has also proved the following theorem.

THEOREM 19. The exponent of any cyclic normal division algebra $D$ of degree $m$ over $F=R(\theta)$ is $m$. (HASSE.)

Let $D$ and $B$ be any two cyclic normal division algebras of the same degree $p$, a prime, over their common centrum $F=R(\theta)$, an algebraic field. Then $D \times B$ is a normal simple algebra so that $D \times B=M \times C$, where $M$ is a total matric algebra and $C$ is a normal division algebra over $F$. Now $(D \times B)^{p}=D^{p} \times B^{p}$ is a total matric algebra by Theorem 18 . Hence $(M \times C)^{p}$ is a total matric algebra so that $C^{p}$ is a total matric algebra and the exponent of $C$ is at most $p$. By Theorem 17 the algebra $M \times C$ and hence $C$ is a cyclically representable algebra. By Theorem 16 algebra $C$ is a cyclic algebra. By Theorem 19 the degree of $C$ is its exponent so that the degree of $C$ is at most $p$. But the degree of $M \times C$ is $p^{2}$ so that the degree of $M$ is at least $p$ and $D \times B$ is not a division algebra.

Lemma. The direct product of any two cyclic normal division algebras of the same degree $p$, a prime, is not a division algebra. 
Let next $D$ and $B$ be any normal division algebras of degrees $p^{e}$ and $p^{f}$ respectively, $p$ a prime and both $e$ and $f$ not zero, over a common centrum $F=R(\theta)$. We may extend the centrum $F$ of $D$ and $B$ to be $Z$, by Theorem 12 , such that $D^{\prime}$ over $Z$ has the property of Theorem 13, and this extension may be obviously made so that simultaneously $B^{\prime}$ has the property of Theorem 13 . If $D \times B$ is a normal division algebra, then, since the order of $Z$ with respect to $F$ is prime to $p$, by Theorem 8 , algebra $D^{\prime} \times B^{\prime}$ over $Z$ is a normal division algebra. Let $y$ in $D$ and $\eta$ in $B$ be the quantities of Theorem 13. Then the algebra $C$ of all quantities of $D^{\prime}$ commutative with $y$ is a cyclic normal division algebra of degree $p$ over its centrum $Z(y)$. The algebra $E$ of all quantities of $B$ commutative with $\eta$ is a cyclic normal division algebra of degree $p$ over its centrum $Z(\eta)$. The quantity $y$ is in the normal division algebra $D^{\prime} \times B^{\prime}$ and the algebra of all quantities of $D^{\prime} \times B^{\prime}$ commutative with $y$ is evidently $C \times B$ over its centrum $K=Z(y)$. In $C \times B$ over $K$ the algebra of all quantities of this algebra commutative with $\eta$ is evidently $C \times E$ over its centrum $K(\eta)$. But $C$ and $E$ are cyclic algebras of degree $p$ over $K(\eta)$, an algebraic field over $R$ and by our Lemma not a division algebra. This is a contradiction of the fact that every sub-algebra of a division algebra is a division algebra. Hence $D \times B$ is not a division algebra.

Now let $D$ and $B$ be any normal division algebras of degrees $m$ and $r$ respectively over $F=R(\theta)$. If $m$ and $r$ have a prime factor $p$ in common, then $D=D_{1} \times D_{2}, B=B_{1} \times B_{2}$, where $D_{1}$ is a normal division algebra of degree $p^{e}$, the highest power of $p$ dividing $m, B_{1}$ is a normal division algebra of degree $p^{f}$, the highest power of $p$ dividing $r$. But $D \times B=\left(D_{1} \times B_{1}\right) \times\left(D_{2} \times B_{2}\right)$, while $D_{1} \times B_{1}$ is not a division algebra as we have proved. Hence $D \times B$ is not a division algebra. Conversely let the orders of $D$ and $B$ be relatively prime. By Theorem 11 , algebra $D \times B$ is a normal division algebra. Passing from degrees to orders, we have the following result.

TheOREM 20. $A$ direct product of two normal division algebras over the same centrum $R(\theta)$ is a division algebra if and only if the orders of the two algebras are relatively prime.

The University of Chicago 